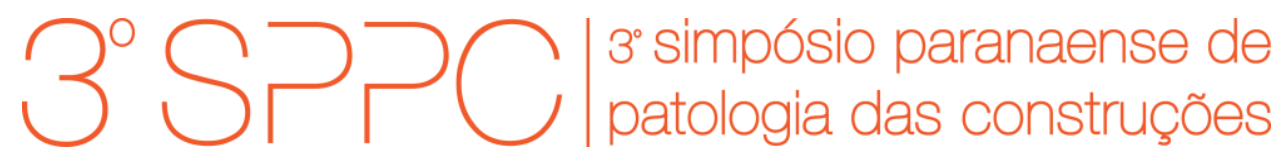

ISSN 2526-7248 artigo n. 3SPPC1022, pp. 245-256, 2018

\title{
Aplicação de modelagem matemática para fins de previsão de vida útil de estruturas de concreto sujeitas à ação de $\mathrm{CO}_{2}$
}

\author{
Rigo, Eduardo"; Oliveira, Carlos Eduardo de ${ }^{2}$; Possan, Edna ${ }^{3}$. \\ ${ }^{1}$ Mestrando, Universidade Federal da Integração Latino-Americana, \\ eduardorigo.e@gmail.com \\ ${ }^{2}$ Mestrando, Universidade Federal da Integração Latino-Americana, \\ eduardo.pbr@gmail.com \\ ${ }^{3}$ Doutora, Universidade Federal da Integração Latino-Americana, \\ epossan@gmail.com
}

\begin{abstract}
Resumo: Um dos principais causadores da corrosão do aço imerso no concreto é o fenômeno conhecido como carbonatação, no qual o $\mathrm{CO}_{2}$ disponível na atmosfera reage com o hidróxido de cálcio presente na matriz cimentícia, transformando-o em carbonato de cálcio e água, acidificando o $\mathrm{pH}$ da estrutura e facilitando a corrosão das armaduras. Um dos meios de evitar tal ocorrência consiste em determinar corretamente a espessura do cobrimento do concreto da estrutura. Para tal, pode-se recorrer a estudos de previsão da carbonatação ao longo do tempo, empregando-se para tal modelos matemáticos de degradação. Tendo em vista que existem vários modelos na literatura que podem ser empregados para tal, este estudo compara a estimativa da profundidade de carbonatação do concreto ao longo do tempo por dois dos mais atuais modelos da literatura. A avaliação dos modelos foi conduzida a partir de um banco de dados da literatura, através da simulação em planilhas do Excel, avaliando-se o erro médio entre o valor estimado e o valor obtido. Os resultados indicam que os modelos analisados apresentam resultados similares, em grande parte dos dados, para a estimava da profundidade de carbonatação do concreto para cimentos isentos de adições. Espera-se que o presente trabalho sirva de contribuição sobre a importância da utilização desses modelos em estruturas de concreto, visto que, dentro de suas limitações, demonstram eficiência em prever a durabilidade e vida útil de concretos nos ambientes em que estão situados, de modo a evitar manifestações patológicas, auxiliando na tomada de decisão.
\end{abstract}

Palavras-chave: carbonatação, vida útil, durabilidade, previsibilidade.

Abstract: One of the main causes of corrosion on reinforced concrete structures is the phenomenon known as carbonation, in which the available atmosphere $\mathrm{CO}_{2}$ reacts with calcium hydroxide present in the cement matrix, transforming it into calcium carbonate and water, acidifying the structure $\mathrm{pH}$ and facilitating the reinforcement corrosion. An alternative to avoid such occurrence is to determine the sufficient thickness of concrete cover. For this, the carbonation depth can be estimated over time using mathematical degradation models. Considering the several models in the literature that can be used for this, this study compares the concrete carbonation depth estimation by two of the most current models of the literature The models evaluation was conducted from a literature database through simulation in Excel spreadsheets, evaluating the average error between estimated value and obtained value. The results indicate that the analyzed models present similar results, in most of the data, for estimating the concrete carbonation depth for free additions cements. It is hoped that the present work contributes about the importance of these models use in concrete structures, because, even with their limitations, they demonstrate efficiency in durability and service life predicting of concretes in their located environment, in order to avoid pathological manifestations, helping in decision making.

Keywords: carbonation, service life, durability, predictability. 
RIGO, E.; OLIVEIRA, C. E.; POSSAN, E., APLICAÇÃO DE MODELAGEM MATEMÁTICA PARA FINS DE PREVISÃO DE VIDA ÚTIL DE ESTRUTURAS DE CONCRETO SUJEITAS À AÇÃO DE CO2. $3^{\circ}$ Simpósio Paranaense de Patologia das Construções (30 SPPC), artigo 3SPPC1022, pp. 245 - 256, 2018. DOI: 10.4322/2526-7248.022

\section{Introdução}

Existe uma grande preocupação relacionada à degradação prematura de estruturas de concreto, ocasionadas principalmente pela corrosão das armaduras embutidas no concreto. De acordo com Tian et al.[1], a taxa de corrosão do aço em estruturas de concreto armado é um dos parâmetros mais importantes para avaliar a sua durabilidade. Durante a sua vida útil, uma estrutura deve ser capaz de suportar ações externas, como, por exemplo, o clima, sendo duráveis suficientes para evitar gastos desnecessários com reparos fora do plano de manutenção [2].

De acordo com Helene [3], aproximadamente 15\% das manifestações patológicas encontradas em estudo realizado na Espanha relacionam-se à corrosão de armaduras. Ainda, de acordo com Stefanoni, Angst e Elsener [4], o risco de corrosão do aço em concretos armados aumenta acentuadamente em condições de usabilidade de muitas estruturas, tais como alta umidade relativa e, especialmente, ciclos de molhagem e secagem.

O fenômeno chamado carbonatação é um dos responsáveis por causar a despassivação da armadura e, consequentemente, degradação e redução da vida útil da estrutura. A carbonatação consiste na reação do dióxido de carbono $\left(\mathrm{CO}_{2}\right)$ presente na atmosfera com os hidróxidos - principalmente com o hidróxido de cálcio $\left(\mathrm{Ca}(\mathrm{OH})_{2}\right)$ - do concreto, formando Carbonato de Cálcio $\left(\mathrm{CaCO}_{3}\right)$ e água, acidificando o pH da estrutura, proporcionando condições ideais para a corrosão de armaduras [5,6]. O avanço da frente de carbonatação está diretamente envolvido com a facilidade da difusão de $\mathrm{CO}_{2}$ pelo cobrimento do concreto $(x)$ em um período de tempo $(t)[7]$.

A carbonatação é potencializada por diversos fatores. Um exemplo é a relação água cimento (a/c). Torgal et al. [8] verificou que em um concreto com relação água/cimento de 0,6 submetido ao efeito de carbonatação, alcançou-se a profundidade de $15 \mathrm{~mm}$ depois de 15 anos, sendo que sob efeito da mesmo teor de carbonatação, um concreto com relação menor (relação a/c $=0,45$ ) precisaria 100 anos para alcançar essa profundidade. Logo, a compreensão das taxas de corrosão em concreto carbonatado, de acordo com a composição de seus componentes, é de grande importância para garantir vida útil longa e livre de manutenções corretivas [3].

A previsibilidade da ocorrência da reação química, bem como a profundidade carbonatada de um concreto pode auxiliar na tomada de decisão. Essa previsibilidade é verificada por meio da aplicação de modelos de profundidade de carbonatação.

Existem 4 tipos de modelos na literatura: empíricos, os quais são baseados em medições experimentais; estatísticos, baseados na aplicação de regressões de múltiplas variáveis e ajustes de funções matemáticas padrões ou idealizadas; numéricos, os quais aplicam abordagens computacionais, dependendo de softwares para análises de dados; e modelos de simulação, baseados na aplicação de redes neurais artificiais (RNA's), que consiste, resumidamente, na aplicação de parâmetros de entrada em um algoritmo específico, gerando dados de saída, que são os resultados esperados [9].

A criação e/ou utilização de modelos de profundidade de carbonatação existentes visa estimar a ocorrência de frentes de carbonatação, bem como a sua 
RIGO, E.; OLIVEIRA, C. E.; POSSAN, E., APLICAÇÃO DE MODELAGEM MATEMÁTICA PARA FINS DE PREVISÃO DE VIDA ÚTIL DE ESTRUTURAS DE CONCRETO SUJEITAS À AÇÃO DE CO2. $3^{\circ}$ Simpósio Paranaense de Patologia das Construções (30 SPPC), artigo 3SPPC1022, pp. 245 - 256, 2018. DOI: 10.4322/2526-7248.022

profundidade, em um concreto inserido em determinadas condições ambientais, sendo uma das ferramentas essenciais para prever a durabilidade e promover a extensão da vida útil de estruturas de concreto armado [7].

De acordo com Köliö et al [2], o pressuposto básico dos principais modelos de carbonatação é a homogeneidade do concreto. Ou seja, as propriedades que determinam a taxa de carbonatação são semelhantes em todas as profundidades de concreto. Porém, na prática, as estruturas de concreto não são idealmente homogêneas, e sua consistência é dependente do tempo necessário de compactação e cura e, especialmente, das condições de umidade ambientais.

Os primeiros modelos de previsão de profundidade de carbonatação surgiram em meados da década de 60 . Até os dias atuais, muitos modelos existentes foram aprimorados, além do surgimento de modelos mais modernos, e considerando variáveis que antes eram negligenciadas. Porém, dúvidas quanto ao resultado final desses modelos perduram quanto a possíveis inconsistências teóricas, tendo como consequência resultados com grande variância entre os modelos [10].

Neste sentido, o objetivo do presente artigo é comparar dois modelos de carbonatação existentes na literatura, avaliando quanto às variáveis envolvidas, aplicabilidade, deficiências, proximidade do valor obtido pelo modelo versus valor experimental, entre outros.

\section{Metodologia}

O presente estudo emprega a simulação como estratégia de pesquisa, tendo como abordagem a pesquisa quantitativa. Primeiramente, fez-se a seleção de modelos matemáticos da literatura para a estimativa da profundidade de carbonatação do concreto, seguido da criação de um banco de dados com base nos trabalhos existentes na literatura.

\subsection{Seleção dos modelos para estimativa de profundidade de carbonatação do concreto}

Por meio de pesquisa bibliográfica foram encontrados na literatura 19 diferentes modelos destinados à estimativa da profundidade de carbonatação do concreto ao longo do tempo, os quais estão descritos em detalhe nos trabalhos de Carmona [9], Pauletti [11], Possan [7], Possan et al. [12], Possan, Dal Molin e Andrade [13], Ta et al. [14] e Ekolu [9]. Para análise comparativa no presente estudo, foram selecionados os modelos propostos por Ekolu [9] e Possan [7], uma vez que apresentam variáveis de entrada de fácil obtenção, os quais são apresentados em detalhe na sequência.

\subsubsection{Modelo de Ekolu [9].}

O modelo de Ekolu [9] estima a profundidade de carbonatação a partir de uma série de equações matemáticas, aplicadas em sequência. $O$ modelo do presente autor parte de uma base de 10 anos de estudos em carbonatação do concreto para a determinação das constantes, considerando 163 conjuntos de dados obtidos no período. 
RIGO, E.; OLIVEIRA, C. E.; POSSAN, E., APLICAÇÃO DE MODELAGEM MATEMÁTICA PARA FINS DE PREVISÃO DE VIDA ÚTIL DE ESTRUTURAS DE CONCRETO SUJEITAS À AÇÃO DE CO2. $3^{\circ}$ Simpósio Paranaense de Patologia das Construções (30 SPPC), artigo 3SPPC1022, pp. 245 - 256, 2018. DOI: 10.4322/2526-7248.022

Levou-se em conta que a ocorrência de carbonatação dependia da umidade relativa do ambiente, da concentração de $\mathrm{CO}_{2}$, da temperatura ambiente, da proteção da estrutura e incidência de chuvas, da qualidade do concreto quanto à mistura, materiais e fatores de processo do concreto, do tipo de cimento e da presença de fissuras e tratamentos superficiais, da cura do concreto e da utilização de agregados especiais e/ou reciclados.

De modo geral, o modelo de Ekolu [9], é representado pela Eq.1.

$$
y=e_{h} \cdot e_{s} \cdot e_{c o} \cdot 1000 \cdot F_{c(t)}^{g} \cdot \sqrt{t}
$$

Onde " $y$ " corresponde a profundidade carbonatada $(\mathrm{mm})$, " $e_{h}$ " é o coeficiente relacional referente à umidade relativa, " $e_{s}$ " corresponde ao coeficiente relacional referente à resistência à compressão do concreto, " $e_{c o}$ " é o coeficiente relacional referente à concentração de $\mathrm{CO}_{2}$ no ambiente, " $F_{c(t)}$ " representa a função de crescimento da resistência do concreto, " $f$ " é o tempo de exposição do concreto (anos) e " $g$ " é o fator dependente do tipo de cimento.

Para determinação do coeficiente "e ${ }_{\mathrm{h}}$ ", utiliza-se a Eq.2:

$$
e_{h}=16 \cdot\left(\frac{R H-35}{100}\right) \cdot\left(1-\frac{R H}{100}\right)^{1,5} \rightarrow 50 \% \leq R H \leq 80 \%
$$

Onde " $R H$ " corresponde à umidade relativa média do ambiente (\%).

Para determinação do coeficiente " $e_{s}$ ", utiliza-se a Eq.3:

$$
e_{s}=\left\{\begin{aligned}
1,0 & \rightarrow \text { Exposição externa protegida } \\
f_{c 28}{ }^{-0,2} & \rightarrow \text { Exposição externa desprotegida }
\end{aligned}\right.
$$

Onde $f_{c 28}$ corresponde à resistência à compressão do concreto aos 28 dias de cura, em MPa.

Para determinação do coeficiente $e_{c o}$, utiliza-se a Eq.4, inserindo, caso necessário, a resistência à compressão do concreto aos 28 dias de cura $\left(f_{c 28}\right)$ e os respectivos coeficientes de correção " $\alpha$ " e " $r$ " da Tabela 1:

$$
e_{c o}=\left\{\begin{array}{c}
\alpha f_{c 28}^{r} \rightarrow 20<f_{c}<60 M P a \\
1,0 \rightarrow f_{c}>60 M P a
\end{array}\right.
$$

Tabela 1: Coeficientes para a equação de " $e_{c o}$ " [9]

\begin{tabular}{cccccc}
\hline \multirow{2}{*}{ Coeficientes } & \multicolumn{5}{c}{ Concentração de $\mathbf{C O}_{2}$ da atmosfera (ppm) } \\
\cline { 2 - 6 } & $\mathbf{2 0 0}$ & $\mathbf{3 0 0}$ & $\mathbf{5 0 0}$ & $\mathbf{1 0 0 0}$ & $\mathbf{2 0 0 0}$ \\
\hline$\alpha$ & 1,40 & 1,00 & 2,50 & 4,50 & 14,00 \\
$r$ & $-0,25$ & 0,00 & $-0,25$ & $-0,40$ & $-0,67$ \\
\hline
\end{tabular}

A determinação do coeficiente $F_{c(t)}$ é obtido através da Eq.5, sendo que seus coeficientes "a" e " $b$ " (Tabela 2) dependem dos valores de resistência do concreto considerados $\left(f_{c 28}\right.$, ao se considerar resistência do concreto aos 28 dias e $f_{c b n}$, ao se considerar a resistência do concreto a longo prazo), bem como as idades do concreto considerado. 
RIGO, E.; OLIVEIRA, C. E.; POSSAN, E., APLICAÇÃO DE MODELAGEM MATEMÁTICA PARA FINS DE PREVISÃO DE VIDA ÚTIL DE ESTRUTURAS DE CONCRETO SUJEITAS À AÇÃO DE CO2. $3^{\circ}$ Simpósio Paranaense de Patologia das Construções (30 SPPC), artigo 3SPPC1022, pp. 245 - 256, 2018. DOI: 10.4322/2526-7248.022

$$
F_{c(t)}=\frac{t}{a+b t} \cdot f_{c} \rightarrow f_{c}=f_{c 28} \text { ou } f_{c b n}
$$

Tabela 2: Coeficientes para a equação de " $F_{c(t)}$ " [9]

\begin{tabular}{cccc}
\hline Resistência à compressão & Idade do concreto & $\boldsymbol{a}$ & $\boldsymbol{b}$ \\
\hline \multirow{2}{*}{$f_{c 28}$} & $\mathrm{t}<6$ anos & 0,35 & $0,6-\mathrm{t}^{0,5} / 50$ \\
& $\mathrm{t} \geq 6$ anos & $0,15 \mathrm{t}$ & $0,5-\mathrm{t}^{0,5} / 50$ \\
\multirow{2}{*}{$f_{c b n}$} & $\mathrm{t}<15$ anos & 0,35 & $1,15-\mathrm{t}^{0,6} / 50$ \\
& $\mathrm{t}>15$ anos & $0,15 \mathrm{t}$ & $0,95-\mathrm{t}^{0,6} / 50$ \\
\hline
\end{tabular}

O coeficiente " $g$ " é determinado de acordo com a Tabela 3, variando de acordo com o tipo de cimento empregado.

Tabela 3: Coeficientes para a equação de " $g$ " [9]

\begin{tabular}{ccc}
\hline $\begin{array}{c}\text { Materiais suplementares } \\
\text { do cimento }\end{array}$ & Tipos de cimento* & $\begin{array}{c}\text { Fator de } \\
\text { condutância "g" }\end{array}$ \\
\hline $20 \%$ quaisquer & CEM I, CEM II/A & $-1,5$ \\
$30 \%$ cinza volante & CEM II/B, CEM IV/A & $-1,4$ \\
$50 \%$ escória & CEM III/A & \\
\hline *. Conforme norma britânica BS EN 197-1 [15].
\end{tabular}

\subsubsection{Modelo de Possan [7]}

O modelo de Possan [7] estima a profundidade de carbonatação a partir de uma equação matemática, apoiada por coeficientes tabelados pela autora, o qual baseou-se no ajuste de dados de degradação devido à carbonatação obtidos via conhecimento de especialistas. O modelo foi obtido a partir do acoplamento das equações de comportamento do concreto relatadas na literatura, especialmente a primeira Lei de Fick. Para ajustar os coeficientes e parâmetros do modelo foram empregados 1298 dados obtidos via conhecimento de experts, advindos das contribuições de 12 experts em carbonatação e durabilidade do concreto.

O modelo (ver Eq. 6), foi posteriormente testado a partir das informações de bancos de dados provenientes de investigações experimentais desenvolvidos por outros pesquisadores brasileiros, representando $85 \%$ dos casos testados.

$$
y=k_{c} \cdot\left(\frac{20}{f_{c}}\right)^{k_{f c}} \cdot\left(\frac{t}{20}\right)^{\frac{1}{2}} \cdot\left\{\exp \left[\left(\frac{k_{a d} \cdot a d^{\frac{3}{2}}}{40+f_{c}}\right)+\left(\frac{k_{C O_{2}} \cdot \mathrm{CO}_{2}{ }^{\frac{1}{2}}}{60+f_{c}}\right)-\left(\frac{k_{U R} \cdot(U R-0,58)^{2}}{100+f_{C}}\right)\right]\right\} \cdot k_{c e}
$$

Onde "y" corresponde a profundidade de carbonatação média do concreto $(\mathrm{mm})$, " $f_{c}$ " representa a resistência característica à compressão axial do concreto (MPa), " $k_{c}$ " representa um fator variável referente ao tipo de cimento empregado (Tabela 4), " $k_{f c}$ " é o fator variável referente à resistência à compressão axial do concreto, em função do tipo de cimento utilizado (Tabela 4), " $t$ " é a idade do concreto (anos), "ad" corresponde ao teor de adição pozolânica no concreto (\% em relação à massa 
RIGO, E.; OLIVEIRA, C. E.; POSSAN, E., APLICAÇÃO DE MODELAGEM MATEMÁTICA PARA FINS DE PREVISÃO DE VIDA ÚTIL DE ESTRUTURAS DE CONCRETO SUJEITAS À AÇÃO DE CO2. $3^{\circ}$ Simpósio Paranaense de Patologia das Construções (30 SPPC), artigo 3SPPC1022, pp. 245 - 256, 2018. DOI: 10.4322/2526-7248.022

de cimento), "UR" é umidade relativa média do ambiente $\left(\% \%^{\star} 0,01\right)$, " $k_{a d}$ " é o fator variável referente às adições pozolânicas do concreto (sílica ativa, metacaulim e

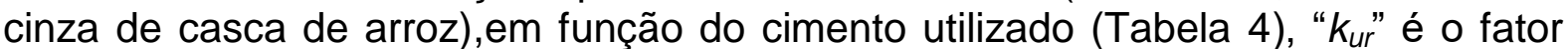
variável referente à umidade relativa, em função do tipo de cimento utilizado; " $\mathrm{CO}_{2}$ " refere-se ao teor de $\mathrm{CO}_{2}$ da atmosfera (\%), " $k_{\mathrm{co}}$ " é o fator variável referente ao teor de $\mathrm{CO}_{2}$ do ambiente, em função do tipo de cimento utilizado (Tabela 4) e " $k_{c e}$ " é o fator variável referente à exposição à chuva, em função das condições de exposição da estrutura (Tabela 5).

Tabela 4: Coeficientes do modelo por tipo de cimento [7]

\begin{tabular}{lccccc}
\hline $\begin{array}{c}\text { Tipo de } \\
\text { cimento } \\
\text { (coeficiente) }\end{array}$ & \begin{tabular}{c} 
Características do concreto \\
\cline { 2 - 6 }$\left(\boldsymbol{k}_{c}\right)$
\end{tabular} & $\begin{array}{c}\boldsymbol{f}_{\boldsymbol{c}} \\
\left(\boldsymbol{k}_{\mathrm{fc}}\right)\end{array}$ & $\begin{array}{c}\text { Adição } \\
\left(\boldsymbol{k}_{\text {ad }}\right)\end{array}$ & $\begin{array}{c}\boldsymbol{C O}_{2} \\
\left(\boldsymbol{k}_{\text {Co2 }}\right)\end{array}$ & $\begin{array}{c}\text { Características do ambiente } \\
\left(\boldsymbol{k}_{\text {UR }}\right)\end{array}$ \\
\hline CP I & 19,8 & 1,7 & 0,24 & 18 & 1300 \\
CP II E & 22,48 & 1,5 & 0,32 & 15,5 & 1300 \\
CP II F & 21,68 & 1,5 & 0,24 & 18 & 1100 \\
CP II Z & 23,66 & 1,5 & 0,32 & 15,5 & 1300 \\
CP III & 30,5 & 1,7 & 0,32 & 15,5 & 1300 \\
CP IV & 33,27 & 1,7 & 0,32 & 15,5 & 1000 \\
CP V ARI & 19,8 & 1,7 & 0,24 & 18 & 1300 \\
\hline
\end{tabular}

Tabela 5: Coeficiente do modelo em função das condições de exposição [7]

\begin{tabular}{lc}
\hline \multicolumn{2}{c}{ Condições de exposição da estrutura } \\
\hline Tipo de exposição & $\boldsymbol{k}_{c e}$ \\
\hline Ambiente interno Protegido da chuva & 1,3 \\
Ambiente externo Protegido da chuva & 1 \\
Ambiente externo Desprotegido da chuva & 0,65 \\
\hline
\end{tabular}

\subsection{Formação do banco de dados}

Para a formação do banco de dados deste estudo, realizou-se uma pesquisa bibliográfica a respeito de estudos sobre carbonatação natural do concreto, uma vez que os modelos selecionados não são aplicados à carbonatação acelerada. Selecionou-se os experimentos de Possan [16], Pauletti [11] e Tasca [17], de modo a aplicá-los no modelos citados no item 2.1. A Tabela 6 apresenta o banco de dados empregado, bem como suas variáveis a serem aplicadas nos modelos.

Um dos principais problemas na formação do banco de dados foi a ausência de algumas variáveis necessárias para a utilização dos modelos, especialmente às relacionado ao ambiente de exposição. Para suprir essa lacuna, alguns valores foram estimados com base nos valores médios indicados para o local de estudo. 
RIGO, E.; OLIVEIRA, C. E.; POSSAN, E., APLICAÇÃO DE MODELAGEM MATEMÁTICA PARA FINS DE PREVISÃO DE VIDA ÚTIL DE ESTRUTURAS DE CONCRETO SUJEITAS À AÇÃO DE CO2. $3^{\circ}$ Simpósio Paranaense de Patologia das Construções (3० SPPC), artigo 3SPPC1022, pp. 245 - 256, 2018. DOI: 10.4322/2526-7248.022

Tabela 6: Banco de dados para aplicação nos modelos $[11,16,17]$

\begin{tabular}{|c|c|c|c|}
\hline \multicolumn{4}{|c|}{ Dados coletados } \\
\hline Propriedades & Possan [16] & Pauletti [11] & Tasca [17] \\
\hline Local de estudo & $\begin{array}{l}\text { Porto Alegre, } \\
\text { RS, Brasil }\end{array}$ & $\begin{array}{l}\text { Porto Alegre, } \\
\text { RS, Brasil }\end{array}$ & $\begin{array}{l}\text { Santa Maria, } \\
\text { RS, Brasil }\end{array}$ \\
\hline Tempo de exposição (anos) & 7 & 2,5 e 4,83 & 4 e 14 \\
\hline Relação água/cimento & 0,80 & 0,40 & 0,55 \\
\hline Tipo de cimento & $\begin{array}{l}\text { CP V ARI } \\
\text { CEM I** }\end{array}$ & $\begin{array}{l}\mathrm{CPI}-\mathrm{S} \\
\mathrm{CEM} \mathrm{I}^{* *}\end{array}$ & $\begin{array}{l}\text { CP V ARI } \\
\text { CEM I** }\end{array}$ \\
\hline $\begin{array}{l}\text { Resistência média à compressão aos } \\
28 \text { dias (MPa) }\end{array}$ & 19,80 & 38,96 & 45,90 \\
\hline Teor de $\mathrm{CO}_{2}$ do ambiente (\%) & 0,035 & 0,071 & $0,035^{\star}$ \\
\hline Umidade relativa (\%) & 70 & $70^{*}$ & $65^{*}$ \\
\hline Tipo de exposição & $\begin{array}{c}\text { Externo } \\
\text { Protegido da } \\
\text { chuva (EP) }\end{array}$ & $\begin{array}{c}\text { Externo } \\
\text { Desprotegido da } \\
\text { chuva (ED) }\end{array}$ & $\begin{array}{c}\text { Interno, sem } \\
\text { controle aq taxa } \\
\text { de } \mathrm{CO}_{2} \text { e UR (I) }\end{array}$ \\
\hline Profundidade média carbonatada (mm) & 12,01 & 1,31 e 2 & 1,41 e 6,35 \\
\hline
\end{tabular}

*: Valores estimados devido à ausência de dados.

**: Tipo de cimento compatibilizado para a utilização no modelo de Ekolu [9].

\section{Resultados e discussão}

A Tabela 7 apresenta as profundidades de carbonatação obtidas a partir do emprego dos modelos de Possan [7] e Ekolu [9], incluindo também dos dados reais e o erro em $\mathrm{mm}$ entre os valores estimados pelos modelos em estudo versus a profundidade real da carbonatação do concreto, para os três banco de dados selecionados [16, 11 e 17].

O modelo de Ekolu não apresenta solução para previsão de profundidade de carbonatação para concretos expostos em ambiente interno, para o qual, segundo a literatura $[4,7,11]$ a carbonatação é mais intensa. Neste sentido, para a simulação com os dados de Tasca [17], considerou-se o ambiente externo protegido da chuva, por esta ser a condição de carbonatação mais próxima ao ambiente interno.

Tabela 7: Dados de profundidade de carbonatação do concreto estimado versus real

\begin{tabular}{|c|c|c|c|c|c|c|}
\hline \multirow{3}{*}{$\begin{array}{l}\text { Fonte dos } \\
\text { dados }\end{array}$} & \multirow{3}{*}{$\begin{array}{l}\text { Idades do } \\
\text { concreto } \\
\text { (anos) }\end{array}$} & \multicolumn{5}{|c|}{ Profundidades de carbonatação $(\mathrm{mm})$} \\
\hline & & \multirow{2}{*}{ Real } & \multicolumn{2}{|c|}{ Modelo de Ekolu [9] } & \multicolumn{2}{|c|}{ Modelo de Possan [7] } \\
\hline & & & Estimado & Erro (\%) & Estimado & Erro (\%) \\
\hline Possan [16] & 7 & 12,01 & 14,78 & $\begin{array}{c}-2,77 \\
(-23,06 \%)\end{array}$ & 10,63 & $\begin{array}{c}1,38 \\
(11,49 \%)\end{array}$ \\
\hline \multirow{2}{*}{ Pauletti [11] } & 2,5 & 1,31 & 1,83 & $\begin{array}{c}-0,52 \\
(-39,69 \%)\end{array}$ & 1,39 & $\begin{array}{c}-0,08 \\
(6,11 \%)\end{array}$ \\
\hline & 4,83 & 2,00 & 2,12 & $\begin{array}{c}-0,12 \\
(-6,00 \%)\end{array}$ & 1,93 & $\begin{array}{c}0,07 \\
(3,50 \%)\end{array}$ \\
\hline \multirow{2}{*}{ Tasca [17] } & 4 & 1,41 & 3,62 & $\begin{array}{c}-2,21 \\
(-156,74 \%)\end{array}$ & 2,77 & $\begin{array}{c}-1,36 \\
(-96,45 \%)\end{array}$ \\
\hline & 14 & 6,35 & 5,67 & $\begin{array}{c}0,68 \\
(10,71 \%)\end{array}$ & 5,18 & $\begin{array}{c}1,17 \\
(18,43 \%)\end{array}$ \\
\hline
\end{tabular}


RIGO, E.; OLIVEIRA, C. E.; POSSAN, E., APLICAÇÃO DE MODELAGEM MATEMÁTICA PARA FINS DE PREVISÃO DE VIDA ÚTIL DE ESTRUTURAS DE CONCRETO SUJEITAS À AÇÃO DE CO2. $3^{\circ}$ Simpósio Paranaense de Patologia das Construções (30 SPPC), artigo 3SPPC1022, pp. 245 - 256, 2018. DOI: 10.4322/2526-7248.022

Avaliando os resultados encontrados, pode-se dizer que ambos os modelos estimaram profundidades de carbonatação similares às reais (ver Figura 2). Como se constata na Figura 1, o erro obtido nas simulações encontra-se distribuído aleatoriamente em torno da média zero, indicando a adequabilidade dos modelos às predições. O erro máximo encontrado foi de $2,77 \mathrm{~mm}$ na aplicação do modelo de Ekolu [9], enquanto que o erro médio (em módulo) foi de 1,26 mm e 0,812 mm para o modelo de Ekolu [9] e Possan [7], respectivamente.

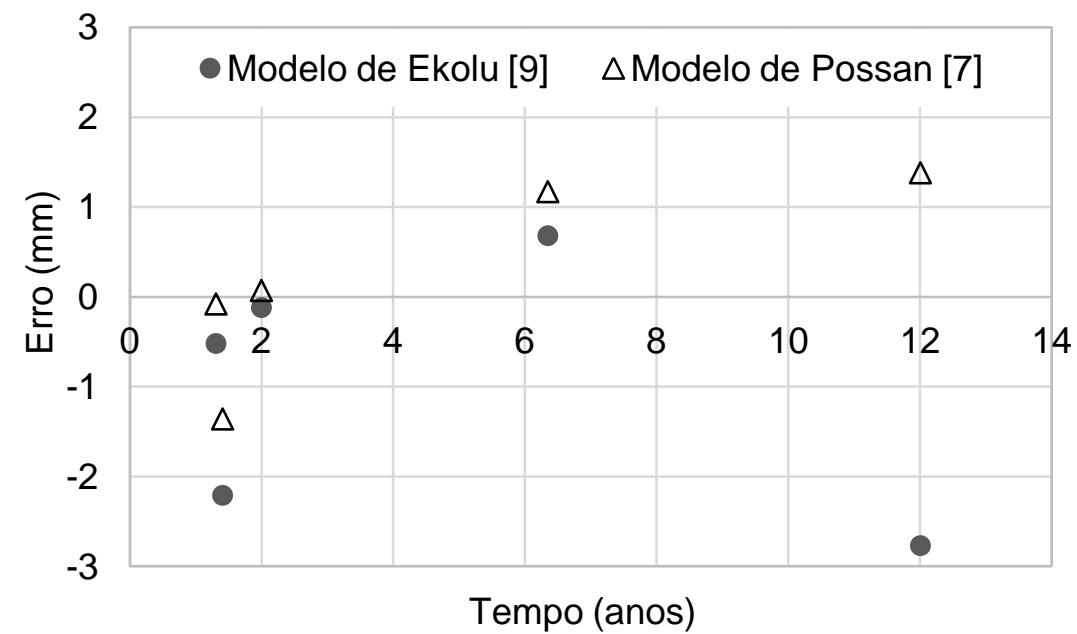

Figura 1: Erro médio das simulações em função do tempo para a profundidade de carbonatação de diferentes concretos $(\mathrm{mm})$

A maior variação do erro se deu para os dados de 4 anos de exposição de Tasca [17], para os quais os dois modelos não puderam representar os dados reais de forma satisfatória, apresentado variações na ordem de $100 \%$. Contudo, o erro da previsão em mm é muito reduzido, sendo de 2,21 $\mathrm{mm}$ para o modelo de Ekolu [9] e $1,36 \mathrm{~mm}$ para o de Possan [7]. Esse fato pode indicar a dificuldade dos modelos predizerem profundidades iniciais de carbonatação, uma vez que os erros associados à medição da profundidade de carbonatação podem interferir nos resultados das simulações. Considerando que a RILEM-CPC-18 [18] indica que profundidades de carbonatação inferiores a $0,5 \mathrm{~mm}$ não são diferenciadas nas medições, os erros encontrados nas simulações realizadas no presente estudo podem ser considerados satisfatórios, indicando que ambos os modelos podem representar a carbonatação de concretos produzidos com cimento isento de adições.

Na Figura 3 e Figura 4 aplica-se os modelos de Ekolu [9] e de Possan [7], respectivamente, para fins de previsão de vida útil ao longo do tempo. Nota-se que para concretos de resistência muito baixa (em torno de $20 \mathrm{MPa}$, em ambiente externo protegido - EP) a profundidade de carbonatação, após 50 anos, será superior a 31,05 mm no modelo de Ekolu [9] e a 28,4 mm no modelo de Possan [7]. Considerando que a NBR 6118 [19] indica cobrimento mínimo de $25 \mathrm{~mm}$ para estruturas em ambiente urbano (classe de agressividade II, moderada), os dois modelos indicam que a estrutura em questão não atingira a vida útil de projeto (VUP). Contudo, ao se elevar a resistência à compressão do concreto para $23 \mathrm{MPa}$, em 50 anos a profundidade de carbonatação será de 24,5 mm (Ekolu [9]) e $22 \mathrm{~mm}$ (Possan [7]), adequando-se ao limite proposto na NBR 6118 [19] e atendendo a VUP da estrutura. Para os concretos com resistência mais elevada (39 e $46 \mathrm{MPa}$ ), 
RIGO, E.; OLIVEIRA, C. E.; POSSAN, E., APLICAÇ̃̃O DE MODELAGEM MATEMÁTICA PARA FINS DE PREVISÃO DE VIDA ÚTIL DE ESTRUTURAS DE CONCRETO SUJEITAS À AÇÃO DE CO2. $3^{\circ}$ Simpósio Paranaense de Patologia das Construções (30 SPPC), artigo 3SPPC1022, pp. 245 - 256, 2018. DOI: 10.4322/2526-7248.022

independente do ambiente de exposição da estrutura (Externo desprotegido - ED, ou Interno - I), verifica-se que a profundidade de carbonatação aos 50 anos seria inferior ao cobrimento mínimo especificado pela NBR 6118 [19], o que é desejável, uma vez que a VUP seria atendida. Com isso, espera-se demostrar a aplicação prática atrelada à utilização e difusão de modelos matemáticos para a estimativa da degradação do concreto para fins de previsão de vida útil.

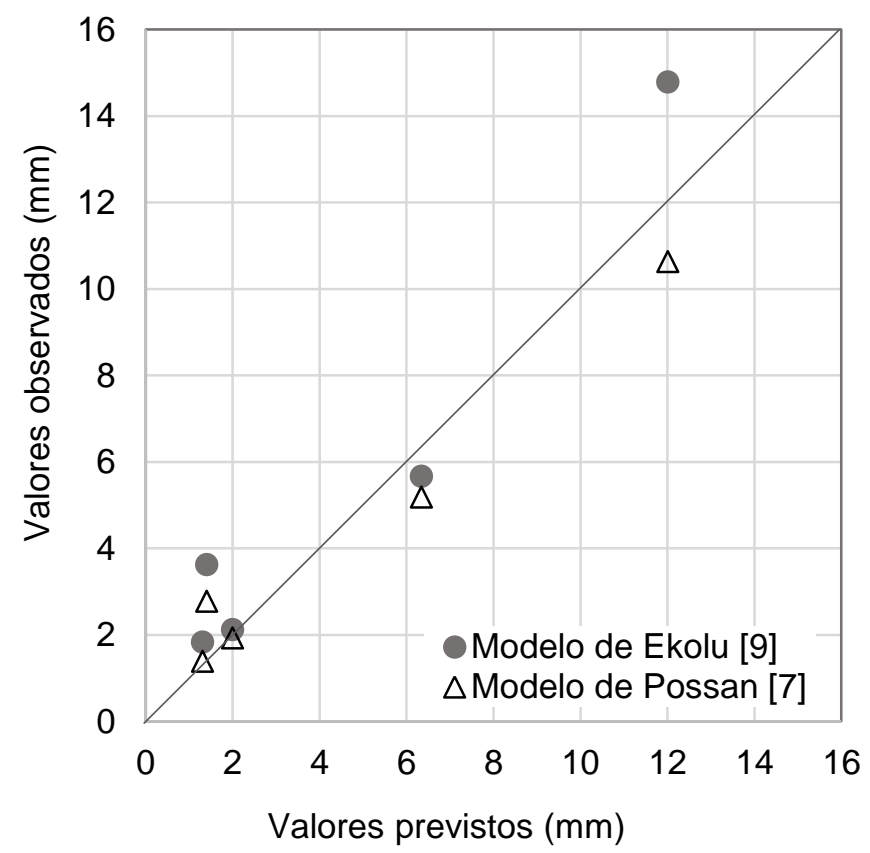

Figura 2: Valores previstos versus valores observados para a profundidade de carbonatação de diferentes concretos $(\mathrm{mm})$

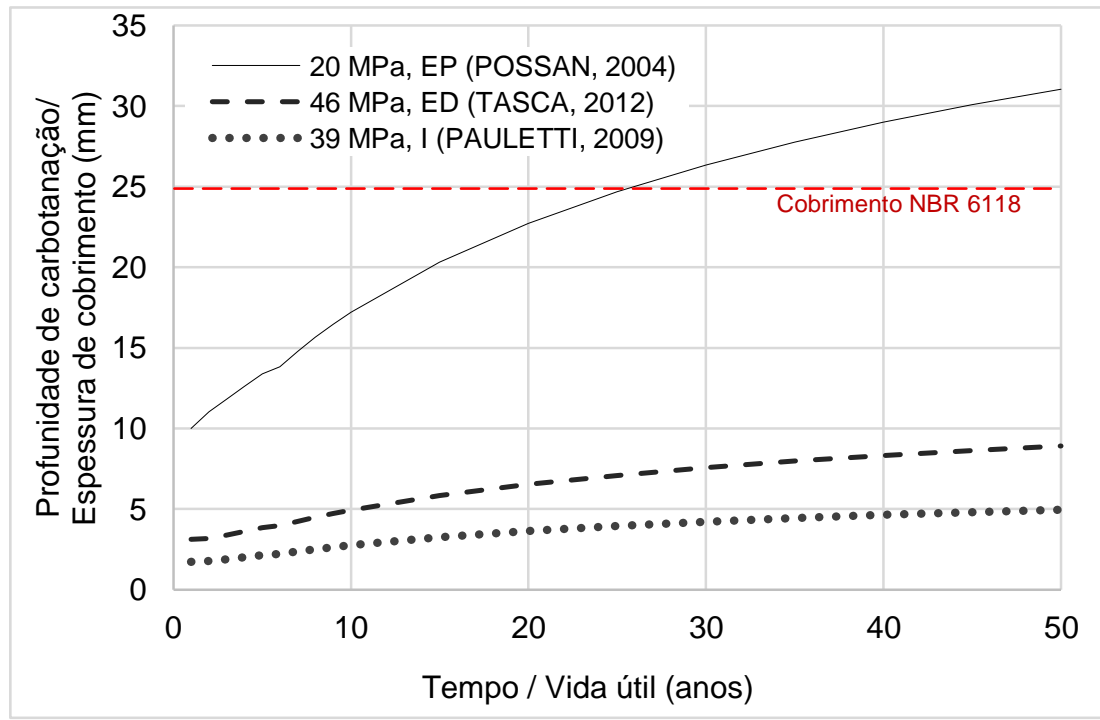

Figura 3: Estimativa de vida útil ao longo do tempo empregando o modelo de Ekolu [9] 
RIGO, E.; OLIVEIRA, C. E.; POSSAN, E., APLICAÇÃO DE MODELAGEM MATEMÁTICA PARA FINS DE PREVISÃO DE VIDA ÚTIL DE ESTRUTURAS DE CONCRETO SUJEITAS À AÇÃO DE CO2. $3^{\circ}$ Simpósio Paranaense de Patologia das Construções (30 SPPC), artigo 3SPPC1022, pp. 245 - 256, 2018. DOI: 10.4322/2526-7248.022

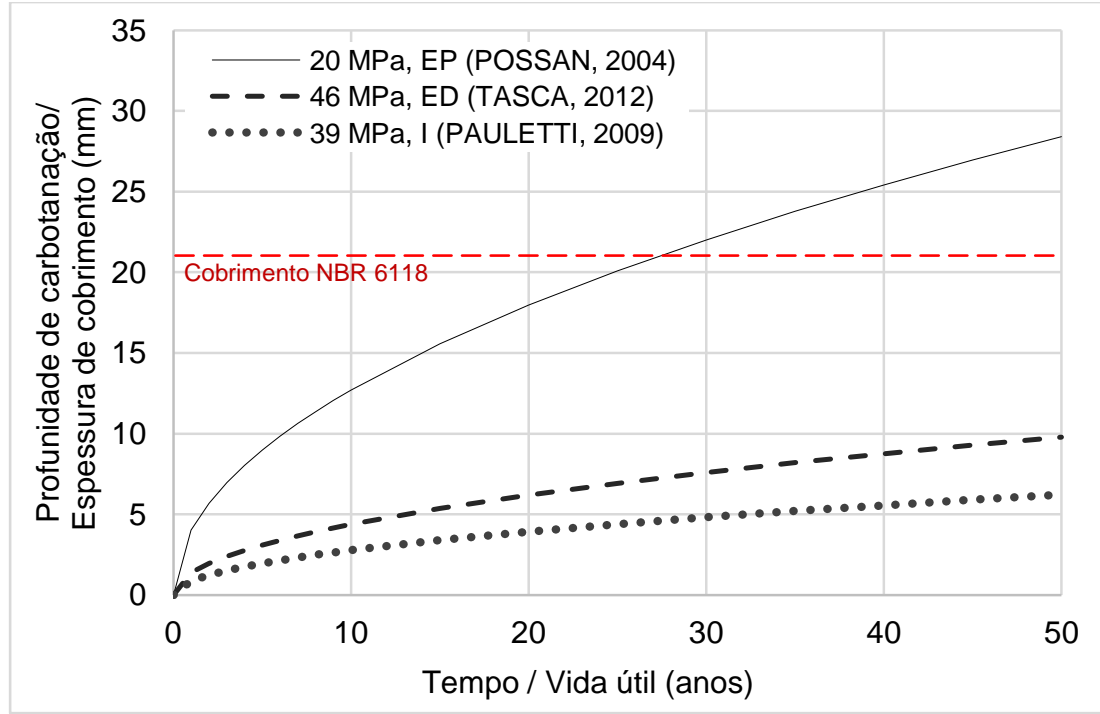

Figura 4: Estimativa de vida útil ao longo do tempo empregando o modelo de Possan [7]

Destaca-se que modelos de estimativa da profundidade de carbonatação podem ser ferramentas úteis para o controle tecnológico de estruturas de concreto, sendo fundamental seu emprego no projeto de estruturas cuja importância (econômica, social e/ou ambiental) é elevada. Diversos modelos de estimativa da profundidade de carbonatação foram propostos pela literatura, de modo que os profissionais da área possam aplicá-los em sua atividade de interesse. Contudo, faz-se necessário avaliar a adequação dos modelos à realidade, sendo prudente comparar as previsões dos mesmos com dados reais de gradação, de modo a identificar o modelo de previsão que melhor representa o fenômeno de carbonatação.

\section{Considerações finais}

Com base nas simulações realizadas no presente estudo pode ser concluir que os modelos de estimativa da profundidade de carbonatação do concreto avaliados (Ekolu [9] e Possan [7]), representaram $80 \%$ dos dados analisados, indicando que os mesmos podem ser empregados em estudos de durabilidade assim como para a previsão de vida útil de projeto (VUP) de estruturas de concreto.

Os dois modelos testados apresentam dados de fácil obtenção, como resistência à compressão aos 28 dias, umidade relativa média e teor de $\mathrm{CO} 2$ do ambiente de exposição, entre outros, o que corrobora para sua aplicação prática.

A diferença entre o dado real e o valor previsto pelos modelos testados (erro médio, em módulo) foi de $1,26 \mathrm{~mm}$ para o modelo de Ekolu [9] e de 0,812 $\mathrm{mm}$ para 0 modelo de Possan [7], o qual é considerado muito baixo para a carbonatação do concreto, indicando o bom desempenho das estimativas para ambos os modelos.

Destaca-se que os modelos de profundidade de carbonatação, desde que respeitadas sua abrangência e limitações de uso, podem ser ferramentais eficazes para o estudo da durabilidade e previsão da vida útil de estruturas de concretos em determinadas condições ambientais, auxiliando a tomada de decisões de projeto e 
RIGO, E.; OLIVEIRA, C. E.; POSSAN, E., APLICAÇÃO DE MODELAGEM MATEMÁTICA PARA FINS DE PREVISÃO DE VIDA ÚTIL DE ESTRUTURAS DE CONCRETO SUJEITAS À AÇÃO DE CO2. $3^{\circ}$ Simpósio Paranaense de Patologia das Construções (30 SPPC), artigo 3SPPC1022, pp. 245 - 256, 2018. DOI: 10.4322/2526-7248.022

evitando futuras manifestações patológicas, tais como a corrosão de armaduras devido à acidificação do meio do concreto devido à carbonatação.

\section{Agradecimentos}

Aos engenheiros civis Cleofas Berwanger e Cláudio Neumann Junior pelas contribuições iniciais ao presente artigo.

\section{Referências}

[1] Tian, Y; Dong, C.; Cheng, X.; Wan, Y.; Wang, G.; Xiao, K.; Li, X. (2017) The micro-solution electrochemical method to evaluate rebar corrosion in reinforced concrete structures, Construction and Building Materials, 40: 607-614.

[2] Köliö, A.; Pakkala, T. A.; Lahdensivu, J.; Kiviste, M. (2014) Durability demands related to carbonation induced corrosion for Finnish concrete buildings in changing climate, Engineering Structures, 62-63: 42-52.

[3] Helene, P (2014). Introdução. In: Sales, A.; Sousa, C.A.C; Almeida, F.C.R.; Cunha, M.P.T.; Lourenço, M.Z. Corrosão em estruturas de concreto armado: Teoria, controle e Método de Análise, 1st ed. Elsevier. Rio de Janeiro

[4] Stefanoni, M.; Angst, U.; Elsener, B. (2018) Corrosion rate of carbon steel in carbonated concrete: A critical review, Cement and Concrete Research, 103: 35-48.

[5] Savija, B.; Lukovic, M (2016) Carbonation of cement paste: Understanding, challenges, and opportunities, Construction and Building Materials, 117:285-301.

[6] Gandía-Romero, J.M.; Campos, I.; Valcuende, M.; García-Breijo, E.; Marcos, M.D.; Payá, J.; Soto, J (2016) Potentiometric thick-film sensors for measuring the $\mathrm{pH}$ of concrete, Cement and Concrete Composites, 68: 66-76.

[7] Possan, E. (2010) Modelagem da carbonatação e previsão de vida útil de estruturas de concreto armado em ambiente urbano. Tese (Doutorado), Universidade Federal do Rio Grande do Sul - UFRGS, Porto Alegre, Brasil.

[8] Torgal, F.P.; Miraldo, S.; Labrincha, J.A.; Brito, J.D (2012) An overview on concrete carbonation in the context of eco-efficient construction: Evaluation, use of SCMs and/or RAC, Construction and Building Materials, 36:141-150.

[9] Ekolu, S. O. (2018) Model for practical prediction of natural carbonation in reinforced concrete: Part 1-formulation, Cement and Concrete Composites, 86:40-56.

[10]Carmona, T.G. (2005) Modelos de previsão de despassivação das armaduras em estruturas de concreto sujeitas à carbonatação. Dissertação (Mestrado), Universidade de São Paulo- USP, São Paulo, Brasil.

[11]Pauletti, C. (2009) Estimativa da carbonatação natural de materiais cimentícios a partir de ensaios acelerados e de modelos de predição. Tese (Doutorado em cotutela), Universidade Federal do Rio Grande do Sul - UFRGS, Porto Alegre, Brasil, Institut National des Sciences Appliquées, Toulouse, França.

[12]Possan, E.; Andrade, J. J. O.; Dal Molin, D. C. C.; Ribeiro, J. L. D. (2011) Modelos matemáticos para estimativa da profundidade de carbonatação do 
RIGO, E.; OLIVEIRA, C. E.; POSSAN, E., APLICAÇÃO DE MODELAGEM MATEMÁTICA PARA FINS DE PREVISÃO DE VIDA ÚTIL

concreto: Estudo comparativo. In: Congresso Brasileiro do Concreto 53IBRACON, Florianópolis, Brasil.

[13]Possan, E.; Dal Molin, D.C.C.; Andrade, J.J.O (2012) Modelos matemáticos para estimativa da carbonatação do concreto. In: Simpósio Internacional sobre Concretos Especiais - 5SINCO, Fortaleza, Brasil.

[14]Ta, V.L.; Bonnet; S.; Kiesse, T.S.; Ventura, A (2016) A new meta-model to calculate carbonation front depth within concrete structures, Construction and Building Materials, 129:172-181.

[15]BS EN 197-1 (2011) Cement - Composition, specifications and conformity criteria for common cements. British Standards Institution - BSI, Londres, Inglaterra.

[16]Possan, E. (2004) Contribuição ao estudo da carbonatação do concreto com adição de sílica ativa em ambiente natural e acelerado. Dissertação (Mestrado), Universidade Federal do Rio Grande do Sul - UFRGS, Porto Alegre, Brasil.

[17]Tasca, M. (2012) Estudo da carbonatação natural de concretos com pozolanas: Monitoramento em longo prazo e análise da microestrutura. Dissertação (Mestrado), Universidade Federal de Santa Maria - UFSM, Santa Maria, Brasil.

[18]RILEM-CPC-18 (1988) Measurement of hardened concrete carbonation depth. TC56-MHM Hydrocarbon Material.

[19]NBR 6118 (2014) Projeto de estruturas de concreto - Procedimento. Associação Brasileira de Normas Técnicas - ABNT, Rio de Janeiro, Brasil. 